\title{
Incidence and risk of hypertension in patients newly treated for multiple myeloma: a retrospective cohort study
}

\author{
Ajai Chari ${ }^{*}$, Khalid Mezzi ${ }^{2}$, Shao Zhu $^{3}$, Winifred Werther ${ }^{4}$, Diana Felici ${ }^{5}$ and Alexander R. Lyon ${ }^{6}$
}

\begin{abstract}
Background: Hypertension is commonly reported in multiple myeloma (MM) patients and may be associated with older age, disease-related complications and consequences of MM treatments. This study evaluated the incidence rates of and risk factors for hypertension and malignant hypertension in newly-treated MM patients in the United States.
\end{abstract}

Methods: Newly-treated adult MM patients were identified from Truven MarketScan claims database from 1/1/05 to $3 / 31 / 14$. Inclusion criteria were new diagnosis of MM with start of MM treatment, $\geq 12$ months continuous enrollment prior to diagnosis, $\geq 30$ days of continuous enrollment following initial diagnosis, and prescription drug coverage. Non-MM patients were matched for age (within $+/-5$ years), sex and distribution of index dates to MM patients. Baseline cardiovascular (CV) comorbidities, incidence rate of hypertension and malignant hypertension in the follow-up period, and risk of hypertension and malignant hypertension based on existing baseline CV comorbidities were evaluated.

Results: A total of 7895 MM patients (38\% with hypertension history) and 23,685 non-MM patients (24\% with hypertension history) were included in the study. Twenty-two percent of MM patients versus $3 \%$ of non-MM patients had baseline renal failure. A higher percentage of MM versus non-MM patients had baseline hypertension in combination with renal failure, congestive heart failure or both. The incidence rate of hypertension in MM and non-MM patients was 260 and 178 per 1000 person-years, respectively. There was a 30\% increase in the risk of hypertension for MM versus non-MM patients: hazard ratio (HR) 1.30 (95\% confidence interval [CI] 1.22, 1.37). In MM patients with a history of hypertension, the risk of malignant hypertension was significantly increased with the following comorbid conditions: cardiomyopathy, HR 2.79 (95\% Cl 1.20, 6.48); renal failure, HR 2.13 (95\% Cl 1.36, 3.34); and diabetes mellitus, HR 1.59 (95\% Cl 1.05, 2.39).

Conclusions: This study confirms that the incidence of hypertension and malignant hypertension is significantly higher in newly-treated MM versus non-MM patients. Hypertension is a risk factor for MM patients developing malignant hypertension. Management of CV comorbidities in MM patients is important based on the increased risk of hypertension and malignant hypertension among patients with these comorbidities.

Keywords: Cardiovascular comorbidity, Hypertension, Incidence, Multiple myeloma, Newly-treated, Risk factor

\footnotetext{
*Correspondence: ajai.chari@mountsinai.org

'Icahn School of Medicine at Mount Sinai, 1 Gustave L. Levy Place, New York,

NY, USA

Full list of author information is available at the end of the article
} International License (http://creativecommons.org/licenses/by/4.0/), which permits unrestricted use, distribution, and reproduction in any medium, provided you give appropriate credit to the original author(s) and the source, provide a link to the Creative Commons license, and indicate if changes were made. The Creative Commons Public Domain Dedication waiver (http://creativecommons.org/publicdomain/zero/1.0/) applies to the data made available in this article, unless otherwise stated. 


\section{Background}

Multiple myeloma (MM) is a bone marrow cancer characterized by clonal plasma cells that may lead to anemia, hypercalcemia, renal insufficiency and bone destruction [1]. It is estimated that 30,330 individuals in the United States (US) will be newly diagnosed with MM in 2016, the majority of whom are elderly ( $50 \%$ aged $\geq 69$ years of age) [2]. Even without MM, the elderly population are at an increased risk for development of cardiovascular (CV)-related comorbidities, including hypertension [3]. Given the mean age at diagnosis, complications of MM (eg, bone pain, renal impairment) and frequent use of corticosteroids (with associated weight gain and anxiety), hypertension and malignant hypertension events are likely to occur in patients undergoing therapy for MM.

Epidemiological data on incidence of hypertension in the general population are available; however, very little is currently published on the incidence rates of hypertensive crisis and malignant hypertension in oncology populations, including patients with MM. Taking into consideration patients with $\mathrm{MM}$ are often elderly and likely to have pre-existing $\mathrm{CV}$ comorbidities and polypharmacy, evaluating the risk factors for hypertension would better enable $\mathrm{CV}$ risk management in these patients. This study evaluates the incidence rates of and risk factors for hypertension and malignant hypertension in newly-treated MM patients in the US.

\section{Methods}

\section{Data source}

This retrospective cohort study utilized data from the Truven MarketScan claims database. This database is representative of healthcare received, including treatment patterns and costs of treatment in more than 36 million privately insured patients across the US. It is a fully integrated, patient-level database containing inpatient, outpatient, drug, laboratory, health risk assessment and benefit design information from patients with commercial and Medicare supplemental insurance. MarketScan is compliant with the Healthcare Information Portability and Accountability Act (HIPAA).

\section{Study population}

The study population consisted of newly-treated patients with MM identified from the Truven MarketScan database between January 1, 2005 and March 31, 2014 using the International Classification of Diseases, Revision 9 (ICD-9) codes 203.0, 203.00, 203.01 or 203.02. Patients were included if they were at least 18 years of age and had newly diagnosed MM (one inpatient or two outpatient claims required) with start of MM treatment, $\geq 12$ months of continuous enrollment prior to the first date of MM diagnosis, $\geq 30$ days of continuous enrollment following initial diagnosis, and prescription drug coverage. To exclude monoclonal gammopathy of undetermined significance and asymptomatic myeloma classified as MM, all patients had to be receiving at least one MM drug identified on prescription claims. Patients were excluded if they had another cancer diagnosis within 12 months prior to, or 12 months following the initial MM diagnosis, and received prior chemotherapy.

A non-MM comparator cohort was identified from the original database which included all claims for the MM cohort. Three randomly selected comparator patients with no MM diagnoses between January 1, 2005 and March 31, 2014 were identified for each of the MM patients so the distribution of index dates for the comparators would match those of the MM patients. Non-MM patients were also matched to MM patients on age (within \pm 5 years of the MM patient's age at index date) and sex. Non-MM patients included in the study were at least 18 years of age, had continuous enrollment during a 12-month baseline plus at least 1-day follow-up time period, and had annual prescription drug coverage during the year (s) included in the 12 months baseline plus at least 1-day follow-up time period. The only exclusion criteria for non-MM patients was having $\mathrm{MM}$; other non-MM cancers were allowed.

\section{Study definitions}

The index date for MM patients was the date of first treatment claim for MM treatment. The index date for non-MM comparator patients was matched to individuals in the MM group with 365 days of continuous enrollment prior to that date. Baseline was defined as the 365 days of continuous enrollment preceding the index date. Followup was defined as the period from index date to first occurrence of an event (first diagnostic code) for those experiencing an event, and was defined as the period from index date to end of enrollment or end of study time period (March 2014) for event-free patients.

\section{Objectives and study measures}

The main objective of this study was to estimate incidence rates of hypertension and malignant hypertension in a representative sample of treated MM patients and non-MM comparator patients in the US. Comparison of hypertensive and malignant hypertensive incidence rates between treated MM patients and non-MM patients, as well as to evaluate the risk of hypertension or malignant hypertension over the follow-up period based on existing hypertension and other baseline CV comorbidities using Cox proportional hazards methods. In addition, the total number of classes of anti-hypertensive medications prescribed at baseline were compared between MM patients and non-MM patients. 
Patient baseline demographics and characteristics

Patient demographics included age, sex, geographic region and calendar year of index date. Hypertensive events were identified from the database using one inpatient or outpatient claim with an ICD-9 code of 401. $\times x$, 402. $\times x$, 403. $\times x, 404 . x \times, 405 . \times x$ or $437.2 \times$. Patients with prior history of hypertension were defined as having a hypertensive event in the baseline period. Other comorbidities included cardiac dysrhythmias, cardiomyopathy, congestive heart failure, ischemic heart disease (acute myocardial infarction and angina), acute myocardial infarction, cerebrovascular disease (hospitalized stroke and transient cerebral ischemia), renal failure, diabetes mellitus, amyloidosis and hyperlipidemia. All comorbidities were identified using one inpatient or outpatient claim (ICD-9 codes; see Additional file 1 Table S1), with the exception of cerebrovascular disease, which was identified using inpatient claims only. The Charlson comorbidity index (CCI) was calculated according to the Quan adaptation [4]. Baseline antihypertensive medications by drug class for treatment of hypertension were identified and included diuretics, angiotensin-converting enzyme inhibitors (ACE-I), angiotensin II blockers, calcium channel blockers and other (alpha blockers, alpha-2 receptor agonists, beta blockers, central agonists, combined alpha and beta blockers, peripheral adrenergic inhibitors, renin inhibitors and vasodilators). Baseline anti-hypertensive medications were defined as treatments prescribed in the 3 months before the index date.

\section{Follow-up period measures}

The hypertensive events were identified as described for the baseline period. Malignant hypertensive events were identified using one inpatient claim with an ICD-9 code of $437.2 \times, 401.0 \times, 402.0 \times, 403.0 \times, 404.0 \times$ or $405.0 \times$. The addition of anti-hypertensive medications in the followup period was compared between MM patient and nonMM patients. For patients with incident hypertension, anti-hypertensive medications were defined as drugs prescribed after hypertension diagnosis.

\section{Statistical analyses}

Incidence rates were estimated using traditional methods and presented per 1000 person-years (PYRs) with a 95\% confidence interval $(\mathrm{CI})$ of any event. A patient was counted in the numerator of the incidence rate at the time of the first diagnostic code for the event in the follow-up period. The risk of hypertension and malignant hypertension (overall and in patients with and without a prior hypertensive event) in the MM and nonMM patients was compared using the Cox proportional hazards regression model. Univariate Cox models were first conducted to assess whether individual baseline variables predicted hypertension or malignant hypertension.
Multivariate Cox models were then applied. Age, sex and geographic region were locked into the model and stepwise methods were used to determine which baseline comorbidities to include in the model. Analyses were conducted using $\mathrm{SAS}^{\circ} 9.3$ (SAS Institute Inc., Cary, NC, USA). Where appropriate, significance was assessed at the $p<0.05$ level.

\section{Results}

A total of 49,565 patients with a MM diagnosis code claim were identified between January 1, 2005 and March 31, 2014 (Fig. 1). Based on inclusion and exclusion criteria, 7895 patients were included in the MM patient cohort for study analysis. A total of 23,685 patients were identified and matched to the MM patients and comprised the non-MM patient cohort for study analysis. The MM and non-MM patients were generally well-matched on distribution of index dates (Table 1).

The baseline demographics and characteristics for MM patients and non-MM patients are shown in Table 1. Both cohorts were equally matched for sex (55.7\% males) and age, with median age (range) at index date of 64 (18-97) years. A total of $49 \%$ of patients were $45-64$ years of age and $47 \%$ were $\geq 65$ years of age ( $47 \%$ ); less than $4 \%$ were $<45$ years of age. The median duration of follow-up for both the MM and non-MM patients was 2 years. The percentage of MM patients with baseline CV comorbidities was higher than that among nonMM patients for each of the comorbidities evaluated (Table 1). Hypertension was the most common comorbidity in both groups, with $38 \%$ of the MM patients (3002/7895) having hypertension at baseline compared with $24 \%$ of the non-MM patients $(5750 / 23,685)$. Heart failure at baseline was observed in 6.7\% (526/7895) and $2.3 \%(549 / 23,685)$ of patients in the MM and non-MM cohorts, respectively. The largest numeric difference between MM and non-MM patients was for presence of baseline renal failure: $22 \%$ of MM patients compared with $3 \%$ of non-MM patients. A total of $13.1 \%$ (1034/7895) of MM patients had both hypertension and acute renal failure at baseline compared with $2.1 \%$ $(494 / 23,685)$ of non-MM patients. A total of $4.1 \%$ of MM patients had both hypertension and congestive heart failure at baseline versus $1.4 \%$ of non-MM patients. The percentage of patients having hypertension, renal failure and congestive heart failure at baseline was $2.3 \%$ for MM patients and $0.5 \%$ for non-MM patients. Ischemic heart disease, diabetes mellitus and hyperlipidemia were present at baseline in $11 \%-18 \%$ of MM patients, with corresponding rates for non-MM patients ranging from $8 \%-16 \%$. Median CCI was 1 (range, 0-12) for the MM patients and 0 (range, 0-15) for the non-MM patients. 


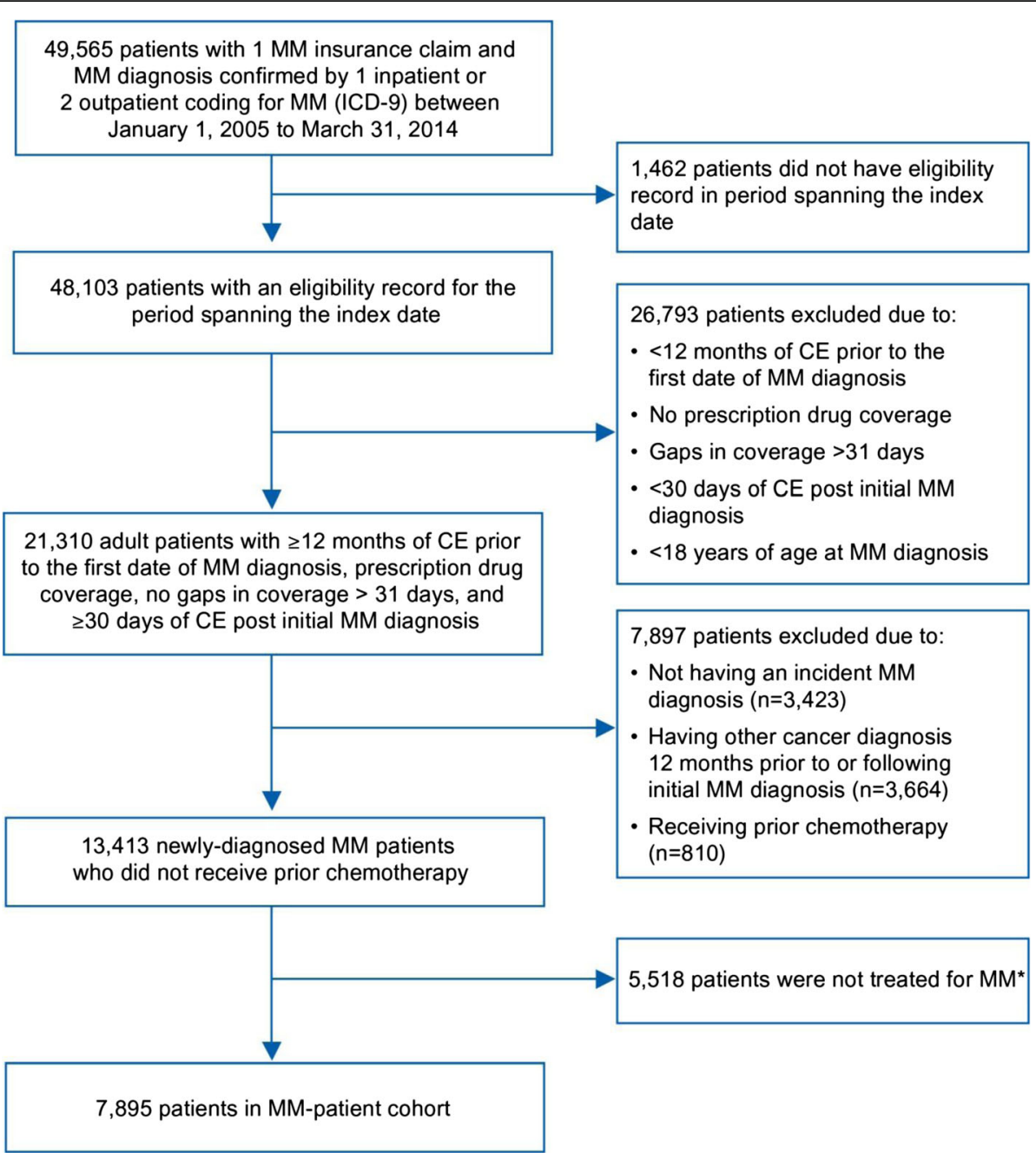

Fig. 1 Selection of MM patient cohort. MM drugs identified on prescription claims. CE, continuous enrollment; ICD-9, international classification of diseases, ninth revision; MM, multiple myeloma

\section{Hypertension in follow-up period}

The incidence rate of hypertension per 1000 PYRs in patients with MM was $260(95 \%$ CI 248, 272) and in non-MM patients was 178 (95\% CI 173, 182) (Fig. 2). Hypertension incidence rates per 1000 PYRs (95\% CI) in $\mathrm{MM}$ and non-MM patients by baseline comorbidities are shown in (Fig. 2).

\section{Malignant hypertension in follow-up period}

The incidence rate per 1000 PYRs (95\% CI) of malignant hypertension in MM-treated patients without a history of hypertension was 3.3 (95\% CI 2.3, 4.5) and in nonMM patients without a history of hypertension was 1.9 (95\% CI 1.5, 2.3). In patients with a prior history of hypertension, the incidence rate per 1000 PYRs (95\% CI) for malignant hypertension in MM-treated patients was $10.3(95 \%$ CI 7.8, 13.2) and in non-MM patients was 4.3
(95\% CI 3.2, 5.5). Rates by baseline comorbidities are shown in (Fig. 3).

\section{Risk of hypertension or malignant hypertension: Cox proportional hazards modeling}

Adjusted hazard ratios (HRs) for hypertension events from the multivariate Cox proportional hazards modeling are presented in Table 2. There was a 30\% increase in the risk of hypertension in MM versus non-MM patients. In patients with baseline ischemic heart disease, renal failure, diabetes or hyperlipidemia, the risk of incident hypertension was significantly increased compared with patients who did not have these comorbidities at baseline $(p<0.001$ for all comparisons; Table 2). Older age ( $\geq 55$ years) also increased the risk of incident hypertension. MM-treated patients with or without a history of hypertension had a significantly higher risk of malignant hypertension during the follow-up period compared with 
Table 1 Baseline demographics and characteristics

\begin{tabular}{|c|c|c|}
\hline Patient characteristics & $\begin{array}{l}\text { MM patients } \\
(n=7895)\end{array}$ & $\begin{array}{l}\text { Non-MM patie } \\
(n=23,685)\end{array}$ \\
\hline \multicolumn{3}{|l|}{ Duration of follow-up, years } \\
\hline Median (range) & $2(0-9)$ & $2(0-10)$ \\
\hline \multicolumn{3}{|l|}{ Age at index date, years } \\
\hline Mean \pm SD & $65.3 \pm 11.8$ & $65.3 \pm 11.8$ \\
\hline Median (range) & $64(18-97)$ & $64(18-97)$ \\
\hline \multicolumn{3}{|l|}{ Age, years, $n(\%)$} \\
\hline $18-34$ & $26(0.3)$ & $78(0.3)$ \\
\hline $35-44$ & $239(3.0)$ & $717(3.0)$ \\
\hline $45-54$ & $1130(14.3)$ & $3390(14.3)$ \\
\hline $55-64$ & $2747(34.8)$ & $8241(34.8)$ \\
\hline $65-74$ & $1812(23.0)$ & $5436(23.0)$ \\
\hline $75+$ & $1941(24.6)$ & $5823(24.6)$ \\
\hline \multicolumn{3}{|l|}{ Sex, n (\%) } \\
\hline Male & $4400(55.7)$ & $13,200(55.7)$ \\
\hline Female & 3495 (44.3) & $10,485(44.3)$ \\
\hline \multicolumn{3}{|l|}{ Year of index date, $n(\%)$} \\
\hline 2005 & $634(8.0)$ & $1591(6.7)$ \\
\hline 2006 & $639(8.1)$ & $1464(6.2)$ \\
\hline 2007 & $607(7.7)$ & $1492(6.3)$ \\
\hline 2008 & $892(11.3)$ & $2154(9.1)$ \\
\hline 2009 & $1136(14.4)$ & $3193(13.5)$ \\
\hline 2010 & $832(10.5)$ & $2445(10.3)$ \\
\hline 2011 & $1007(12.8)$ & $3644(15.4)$ \\
\hline 2012 & $1102(14.0)$ & $4177(17.6)$ \\
\hline 2013 & $908(11.5)$ & $3039(12.8)$ \\
\hline 2014 & $138(1.7)$ & $486(2.1)$ \\
\hline \multicolumn{3}{|l|}{ Comorbidities at baseline, $n(\%)$} \\
\hline Hypertension & $3002(38.0)$ & $5750(24.3)$ \\
\hline Renal failure & $1698(21.5)$ & $696(2.9)$ \\
\hline Hyperlipidemia & $1399(17.7)$ & $3712(15.7)$ \\
\hline Diabetes mellitus & $1242(15.7)$ & $3007(12.7)$ \\
\hline Ischemic heart disease & $841(10.7)$ & $1777(7.5)$ \\
\hline Cardiac dysrhythmias & $563(7.1)$ & $1019(4.3)$ \\
\hline Congestive heart failure & $526(6.7)$ & $549(2.3)$ \\
\hline Cardiomyopathy & $168(2.1)$ & $176(0.7)$ \\
\hline Amyloidosis & $110(1.4)$ & $3(0.01)$ \\
\hline Acute myocardial infarction & $106(1.3)$ & $133(0.6)$ \\
\hline Cerebrovascular disease $^{a}$ & $100(1.3)$ & $159(0.7)$ \\
\hline Hypertension + renal failure & $1034(13.1)$ & $494(2.1)$ \\
\hline $\begin{array}{l}\text { Hypertension + congestive } \\
\text { heart failure }\end{array}$ & $322(4.1)$ & $320(1.4)$ \\
\hline $\begin{array}{l}\text { Hypertension }+ \text { renal failure }+ \\
\text { congestive heart failure }\end{array}$ & $179(2.3)$ & $116(0.5)$ \\
\hline
\end{tabular}

Table 1 Baseline demographics and characteristics (Continued)

$\mathrm{CCl}$

\begin{tabular}{lll} 
Mean \pm SD & $1.44 \pm 1.93$ & $0.41 \pm 1.01$ \\
Median (range) & $1(0-12)$ & $0(0-15)$ \\
\hline
\end{tabular}

CCl: Charlson comorbidity index, MM: multiple myeloma, SD: standard deviation

${ }^{\mathrm{a} B a s e d}$ on inpatient claim only

non-MM patients (previous history of hypertension: HR $1.90,95 \%$ CI 1.26, 2.87, $p<0.01$; no prior history of hypertension: HR 1.54, 95\% CI 1.04, 2.28, $p<0.05)$. In patients with a prior history of hypertension, presence of cardiomyopathy, renal failure or diabetes at baseline significantly increased the risk of malignant hypertension compared with absence of these comorbidities at baseline $(p<0.05$ all comparisons). In patients without a prior history of hypertension, only age $\geq 65$ years versus $18-54$ years and mild or high CCI versus low CCI at baseline was associated with an increased risk of malignant hypertension in the follow-up period ( $p<0.05$ all comparisons).

\section{Anti-hypertensive medications in MM and non-MM patients}

The numbers of MM and non-MM patients taking antihypertensive medications at baseline are shown in Table 3. The proportion of patients receiving at least one class of anti-hypertensive medication at baseline was the same for MM and non-MM patients (71\%). The number of classes of anti-hypertensive medication at baseline between the two groups was similar (Fig. 3). Among patients who were treated for hypertension, the most common medications at baseline for both groups were diuretics, ACE-I, calcium channel blockers and angiotensin II receptor blockers (ARBs) (Table 3). For patients with incident hypertension, 1425 of 1865 (76.4\%) MM patients and 4548 of $5861(77.6 \%)$ non-MM patients received at least one class of anti-hypertensive medication during followup. A total of $16.0 \%$ of MM patients and $10.4 \%$ of nonMM patients received one new class of anti-hypertensive medication during the follow-up period; $9.9 \%$ of MM patients and $11.2 \%$ of non-MM patients received two additional classes of anti-hypertensive medications during the follow-up period (Fig. 4).

\section{Discussion}

Hypertension is commonly reported in patients with MM in clinical trials and may be associated with older age, disease-related complications or consequence of MM treatments [5-7]. However, little is known about the incidence of hypertension or malignant hypertension in the broader population of patients outside of clinical trials. To our knowledge, this is the first study to estimate the incident rates of hypertension and malignant 


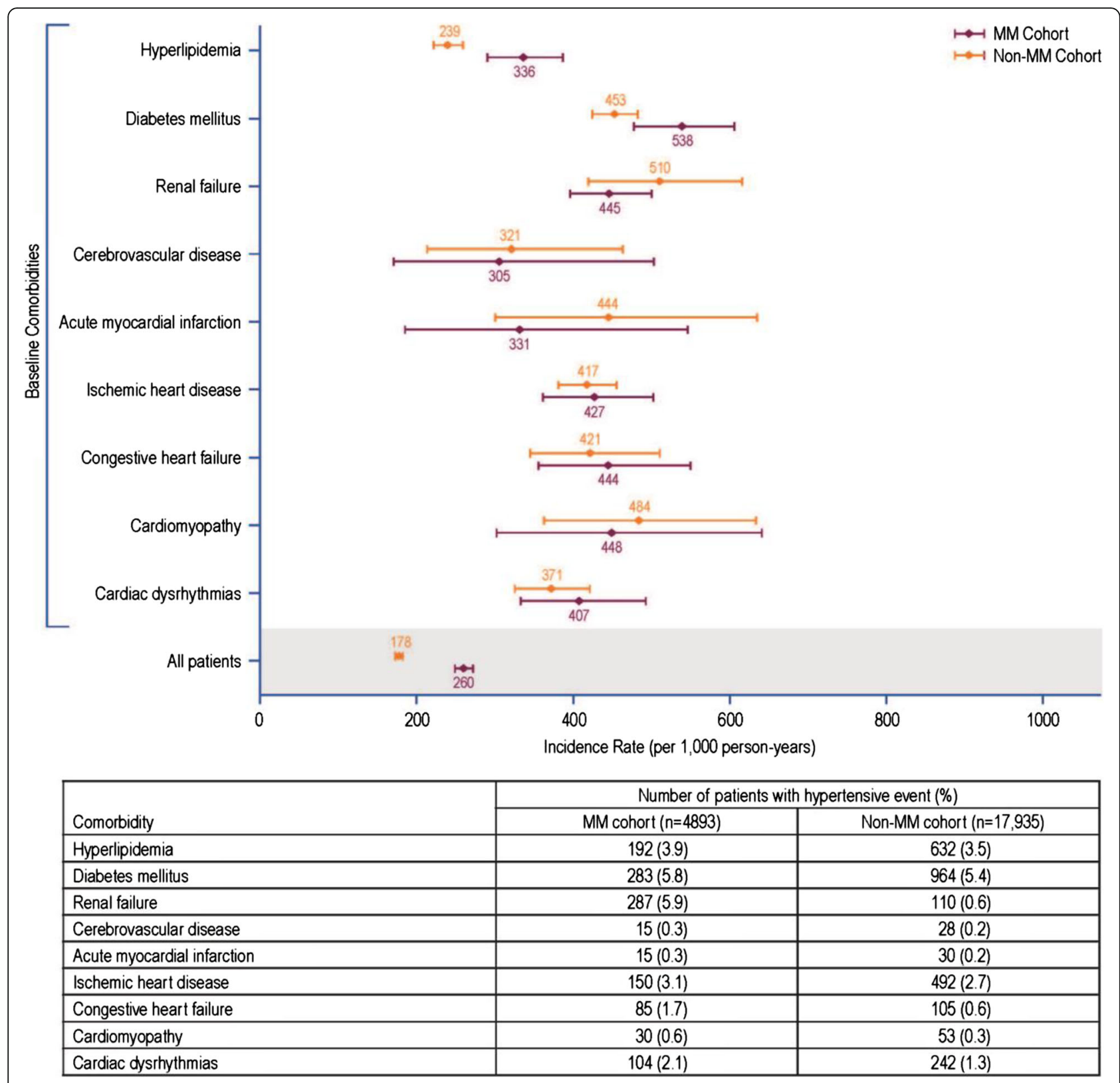

Fig. 2 Incidence rate of hypertension (per 1000 PYRs) and 95\% confidence intervals. MM, multiple myeloma; PYRs, person-years

hypertension in a population of newly-diagnosed, MMtreated patients in the US not participating in clinical trials compared with age- and gender-matched non-MM patients. There is a recent study by Kistler and colleagues that reported incidence rates of hypertension in combination with arterial events as part of their evaluation of cardiac events in MM and non-MM patients based on MarketScan data [8]. They found no significant difference in risk of hypertension/arterial events between $\mathrm{MM}$ and non-MM patients. This differs from the results of this current study which found a 30\% higher risk of hypertension in MM patients versus non-MM patients.
There were a couple of significant differences in study design between the two studies. Kistler et al. did not specifically study hypertension events alone; they evaluated the incidence of hypertensive and arterial events combined. In addition, the MM patients in the Kistler study had a longer duration of MM disease, as the inclusion criteria for the MM cohort required patients to have had at least three anti-myeloma treatments (thereby introducing confounding medical issues such as autonomic or adrenal insufficiency, weight loss, etc.), whereas the current study included newly-diagnosed MM patients with at least one anti-myeloma treatment. 


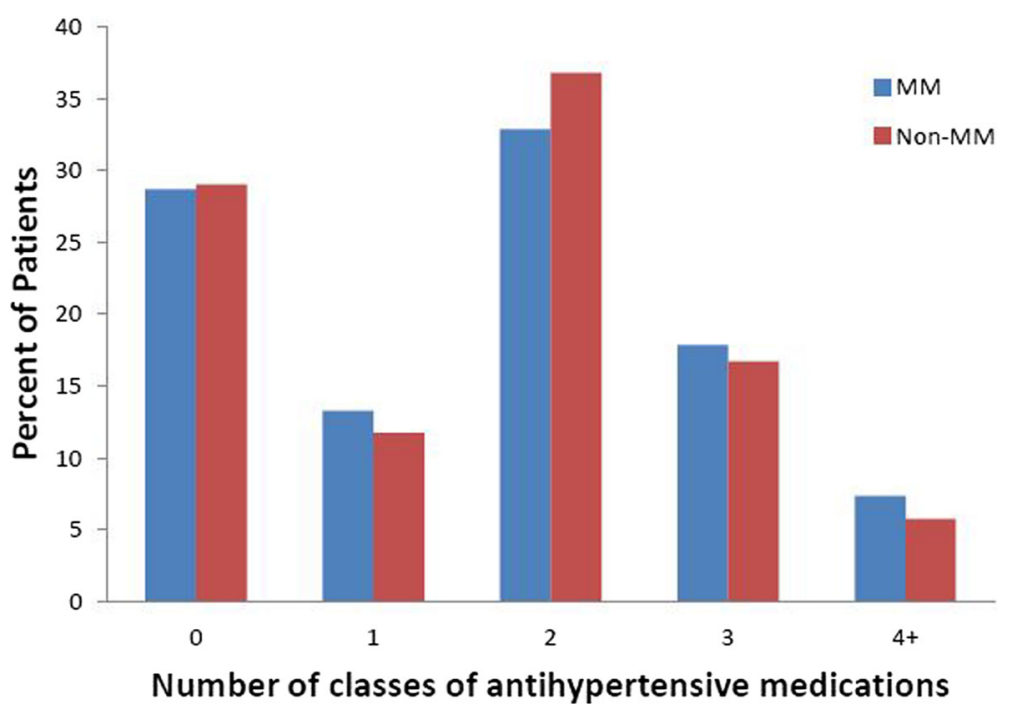

Fig. 3 Number of classes of anti-hypertensive medications at baseline for MM and non-MM patients. Anti-hypertensive medications by class included diuretics, ACE-I, angiotensin II blockers, calcium channel blockers and others (alpha blockers, alpha-2 receptor agonists, beta-blockers, central agonists, combined alpha and beta blockers, peripheral adrenergic inhibitors, renin inhibitors and vasodilators). ACE-I, angiotension-converting enzyme inhibitor; MM, multiple myeloma

In this study, the prevalence rate of hypertension in non-MM patients (33\% of patients) is comparable with published data for the US adult population (1 out of 3 adults) $[9,10]$. That said, the incidence of hypertension and malignant hypertension is significantly higher in newly-treated MM patients compared with non-MM patients. Multi-variate analyses showed that patients with MM had a statistically significant increased risk of hypertension compared with non-MM patients and also

Table 2 Multivariable Cox proportional hazards model: Predictors of hypertension

\begin{tabular}{|c|c|c|c|}
\hline Predictor & Level & HR $(95 \% \mathrm{Cl})$ & $p$-value \\
\hline $\begin{array}{l}\text { Patient cohort } \\
\text { (reference: non-MM patients) }\end{array}$ & MM patients & $\begin{array}{l}1.30 \\
(1.22,1.37)\end{array}$ & $<0.0001$ \\
\hline \multirow[t]{3}{*}{ Age (reference: 18-54 years) } & $55-64$ years & $\begin{array}{l}1.82 \\
(1.69,1.97)\end{array}$ & $<0.0001$ \\
\hline & $65-74$ years & $\begin{array}{l}2.55 \\
(2.36,2.76)\end{array}$ & $<0.0001$ \\
\hline & $75+$ years & $\begin{array}{l}2.88 \\
(2.66,3.11)\end{array}$ & $<0.0001$ \\
\hline \multirow[t]{4}{*}{$\begin{array}{l}\text { Comorbidities at baseline } \\
\text { (yes vs. no) }\end{array}$} & $\begin{array}{l}\text { Ischemic heart } \\
\text { disease }\end{array}$ & $\begin{array}{l}1.29 \\
(1.20,1.40)\end{array}$ & $<0.0001$ \\
\hline & Renal failure & $\begin{array}{l}1.43 \\
(1.27,1.61)\end{array}$ & $<0.0001$ \\
\hline & Diabetes mellitus & $\begin{array}{l}1.72 \\
(1.59,1.86)\end{array}$ & $<0.0001$ \\
\hline & Hyperlipidemia & $\begin{array}{l}1.16 \\
(1.07,1.26)\end{array}$ & $<0.0001$ \\
\hline CCI (reference: low [0]) & Mild $(1,2)$ & $\begin{array}{l}1.11 \\
(1.03,1.20)\end{array}$ & $<0.01$ \\
\hline
\end{tabular}

CCI Charlson comorbidity index, $H R$ hazard ratio, $M M$ multiple myeloma a significantly increased risk of malignant hypertension in both MM patients with or without a history of hypertension compared with non-MM patients. Whether the increased risks of hypertension and malignant hypertension found for MM patients were due to disease-unrelated factors, disease-related comorbidities or a combination of these factors is difficult to determine. Older age and male gender pre-disposes MM patients to an increased risk of hypertension; however, this study controlled for both these factors by using age- and sex-matched non-MM patients.

Results of multi-variable modeling found that the presence of several CV comorbidities increased the risk of hypertension and malignant hypertension in MM patients. In patients without a prior history of hypertension, co-existing ischemic heart disease, renal failure, diabetes and hyperlipidemia increased the risk of hypertension. In patients with a prior history of hypertension, co-existing cardiomyopathy, renal failure or diabetes greatly increased the risk of malignant hypertension. The presence of all of these co-morbidities was significantly higher in the MM population than in the nonMM population at baseline. High levels of CV comorbidities in MM patients have been noted in another non-clinical study of newly-diagnosed MM patients. Chen et al. reported that close to half $(47.9 \%)$ of all newly-diagnosed MM patients $(N=8239)$ identified from commercial medical and Medicare claims databases had more than one type of comorbidity at baseline (6 months prior to MM diagnosis), with $43.9 \%$ of patients having metabolic comorbidities, $21.4 \%$ with CV diseases and $11.5 \%$ with renal conditions [11]. 
Table 3 Baseline anti-hypertensive drugs in patients with a history of hypertension

\begin{tabular}{lll}
\hline & $\begin{array}{l}\text { MM Patients } \\
(n=3002)\end{array}$ & $\begin{array}{l}\text { Non-MM Patients } \\
(n=5750)\end{array}$ \\
\hline $\begin{array}{lll}\text { Anti-hypertensive drug, n (\%) } \\
\text { All drugs }^{\mathrm{a}}\end{array}$ & $2141(71 \%)$ & $4082(71 \%)$ \\
$\quad$ Diuretic & $1704(80 \%)^{\mathrm{b}}$ & $3339(82 \%)^{\mathrm{b}}$ \\
ACE-I & $1113(52 \%)^{\mathrm{b}}$ & $2180(53 \%)^{\mathrm{c}}$ \\
Calcium channel blocker & $1081(50 \%)^{\mathrm{b}}$ & $1773(43 \%)^{\mathrm{c}}$ \\
ARB & $914(43 \%)^{\mathrm{b}}$ & $1723(42 \%)^{\mathrm{c}}$ \\
Any other drugs & $53(2 \%)^{\mathrm{b}}$ & $102(2 \%)^{\mathrm{c}}$ \\
\hline
\end{tabular}

$A C E-$ : : angiotensin-converting enzyme inhibitor, $A R B$ : angiotensin II receptor blocker, $M M$ : multiple myeloma

${ }^{a}$ All anti-hypertensive drugs included diuretics, ACE-I, ARBs, calcium channel blockers and other (alpha blockers, alpha-2 receptor agonists, beta-blockers, central agonists, combined alpha and beta blockers, peripheral adrenergic inhibitors, renin inhibitors and vasodilators)

bercentage derived from $n=2141 \mathrm{MM}$ patients treated for hypertension ${ }^{c}$ Percentage derived from $n=4082$ non-MM patients treated for hypertension

Renal dysfunction is very common in MM patients and renal failure is a negative prognostic factor for patient survival [5]. In this study, renal failure at the start of treatment in patients without a history of baseline hypertension did appear to be a risk factor for hypertension during treatment; however, patients were only at risk for malignant hypertension if renal failure was also associated with baseline hypertension.

Hypertension has been reported to be twice as frequent in patients with diabetes than in those without diabetes [12]. The findings from this study also show close to double the rate of hypertension in non-MM patients with diabetes (54\%) versus those without diabetes
(30\%) (data not shown). For MM patients, the rate of hypertension was $54 \%$ in those with co-existing diabetes and $36 \%$ in patients without diabetes (data not shown). This is of particular concern given that the routine use of corticosteroids in myeloma therapy can lead to new diagnoses of diabetes or worsen glycemic control of those with known diabetes. Taken together, these results show that control and prevention of hypertensive events in $\mathrm{MM}$ patients must include management of $\mathrm{CV}$ comorbidities.

Per the 8th Joint National Committee of 2014 evidencebased guidelines for management of high blood pressure in adults, "hypertension is one of the most important preventable contributors to disease and death" [13]. The guidelines recommend initiating drug treatment in nonblack hypertensive patients with an ACE-I, ARB, calcium channel blocker or thiazide-type diuretic; in black hypertensive patients, initial therapy should include a calcium channel blocker or thiazide-type diuretic. The most common anti-hypertensive medications at baseline for this study were diuretics, ACE-I, calcium channel blockers and ARBs. For patients with a history of hypertension, the same percentage of patients (71\%) in the MM and nonMM groups were receiving anti-hypertensive medications, as well as similar numbers of anti-hypertensive medications at baseline. During the follow-up period, similar percentages were seen between the MM patients and nonMM patients with incident hypertension.

The choice of anti-hypertensive therapy in a myeloma patient, however, must take into account myelomaassociated renal failure (hence caution with diuretics and ACE-I/ARB), hypercalcemia or hyperuricemia (which

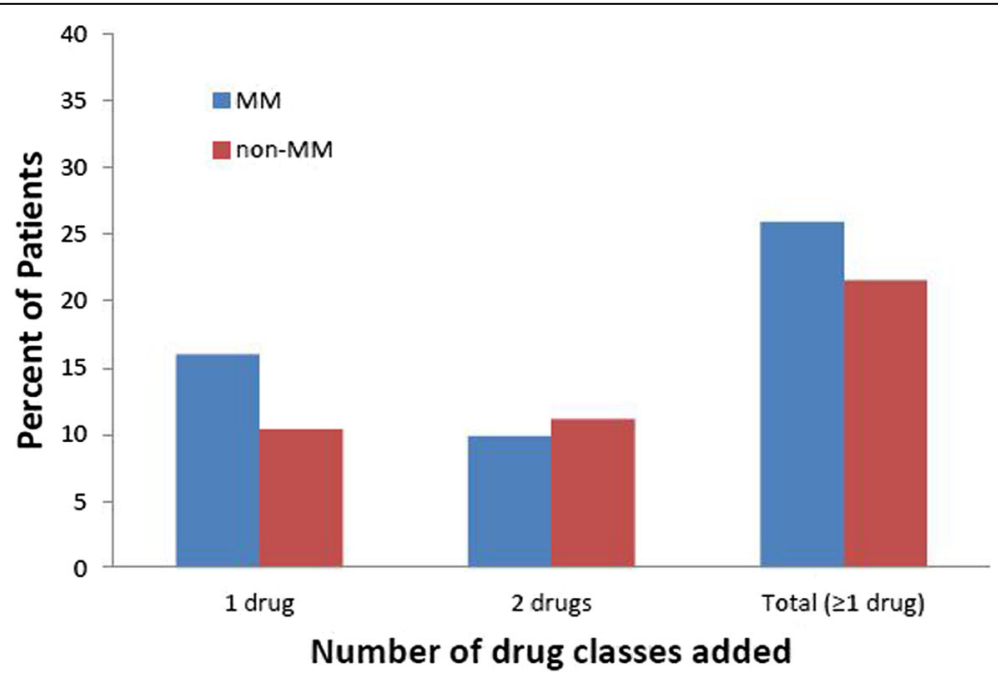

Fig. 4 Addition of anti-hypertensive medications during the follow-up period for MM and non-MM patients. Classes of anti-hypertensive medications added included diuretics, ACE-I, angiotensin II blockers, calcium channel blockers and other (alpha blockers, alpha-2 receptor agonists, beta-blockers, central agonists, combined alpha and beta blockers, peripheral adrenergic inhibitors, renin inhibitors and vasodilators). ACE-l, angiotension-converting enzyme inhibitor; MM, multiple myeloma 
can be exacerbated by thiazides), and steroid-related edema (which can be exacerbated by calcium channel blockers). It should also be noted that grade 3 hypertension in clinical trials does not necessarily equate to markedly elevated blood pressures or malignant hypertension, as the addition of blood pressure medications is considered a grade 3 hypertension adverse event per National Cancer Institute Common Toxicity Criteria definition. Addition of antihypertensive medications was not included as a hypertensive event in this study; however, evaluation of antihypertensive medications in the follow-up period found approximately $6 \%$ more MM patients than non-MM patients had one class of anti-hypertensive medication added.

The results of this study emphasize that $\mathrm{CV}$ and hypertensive adverse effects cannot be evaluated in clinical trials without a comparator arm, given the high incidence rates of these complications in MM patients. MM patients entering the trials are already at a high risk for hypertensive events, and existing hypertension is a major risk factor for development of malignant hypertension. Hypertension has been reported as an adverse event in studies of patients undergoing MM treatment [5-7, 14-18].

Although this study evaluated hypertension and malignant hypertension in MM patients undergoing treatment for $M M$, it did not evaluate results for specific antimyeloma treatments. There are some other limitations of this study. MarketScan claims database better represents the demographic distribution of employed populations while under-representing the elderly, unemployed and disabled. This may be a reason why the median age of MM diagnosis in this study, 65 years, was close to, but a little younger than that published in the literature for median age of MM incidence (69 years SEER cancer statistics) [2]. The MarketScan database does not include information about race, precluding examining the effect of race on these findings (for example, hypertension rates in black vs. white patients, since MM is two-fold more common than in white patients) [2]. In addition, survival data and hypertension and other CV co-morbidity risk factors such as obesity, diet, physical activity and smoking status are not included in the database. There exists the possibility that malignant hypertension may be underestimated in MM patients for whom hospital admissions were not explicitly coded as such, due to the presence of other acute medical issues such as disease progression. Finally, this study could not control for ascertainment bias; MM patients under treatment would be evaluated more frequently by physicians than non-MM patients and thus have a higher probability of hypertensive events reported.

\section{Conclusion}

The incidence of hypertension and malignant hypertension is significantly higher in newly-treated MM patients compared with non-MM patients. Hypertension is a risk factor for MM patients developing malignant hypertension. The presence of hypertension and co-existing cardiomyopathy, renal failure or diabetes also significantly increase the risk of MM patients developing malignant hypertension. Due to the introduction of novel efficacious agents that will likely improve life expectancy, more MM patients will be living longer and will likely be at a greater risk of developing CV complications. The findings of this study highlight the need for a multidisciplinary approach in managing $M M$, especially in elderly patients at a greater risk of $\mathrm{CV}$ events. A close collaboration between oncologists, cardiologists, nephrologists and primary care physicians is warranted.

\section{Additional file}

Additional file 1: Table S1. Comorbidity ICD-9 codes. ICD-9, International Classification of Diseases, ninth revision. (DOCX $25 \mathrm{~kb}$ )

\section{Abbreviations \\ ACE-I: Angiotensin-converting enzyme inhibitor; ARB: Angiotension II receptor blocker; $\mathrm{CCl}$ : Charlson comorbidity index; $\mathrm{Cl}$ : Confidence interval; CV: Cardiovascular; HIPAA: Healthcare Information Portability and Accountability Act; HR: Hazard ratio; ICD-9: International Classification of Diseases, Revision 9; MM: Multiple myeloma; PYR: Person year; SD: Standard deviation; US: United States}

\section{Acknowledgements}

ARL is supported by the National Institute of Health Research Cardiovascular Biomedical Research Unit at the Royal Brompton Hospital and the British Heart Foundation (FS/11/67/28954).

\section{Funding}

Financial support for this study was provided by Amgen, Inc., South San Francisco, CA. Medical writing assistance was provided by BlueMomentum, an Ashfield Company, part of UDG Healthcare PLC, and funded by Amgen, Inc.

Availability of data and materials

The data that support the findings of this study are available from Truven Health Analytics but restrictions apply to the availability of these data, which were used under license for the current study, and so are not publicly available. Data are however available from the authors upon reasonable request and with permission of Truven Health Analytics.

\section{Authors' contributions}

$A C, A L, K M, D F$ and WW: conception and design of the study. DF, SZ and WW: acquisition of data and data analysis. All authors participated in data interpretation, have been involved in drafting the manuscript or revising it critically for important intellectual content, have provided final approval of the version to be published, and agree to be accountable for all aspects of the work.

\section{Competing interests}

AC has served as a consultant for and received research funding from Janssen Pharmaceutical and Bristol-Myers Squibb, and has received research funding and honoraria from Takeda, Celgene, Array BioPharma, Novartis and Onyx Pharmaceuticals. ARL has served as a consultant for Onyx Pharmaceuticals. KM, SZ and WW are employees of Amgen Inc. DF is an employee of Onyx

Pharmaceuticals, Inc., an Amgen subsidiary.

Consent for publication

Not applicable. 


\section{Ethics approval and consent to participate}

Ethics approval was not applicable to this study. Given the de-identified nature of the claims data used in this study, informed consent was not required by HIPAA rules. Reporting of individual person data was not applicable in this study.

\section{Author details}

${ }^{1}$ Icahn School of Medicine at Mount Sinai, 1 Gustave L. Levy Place, New York, NY, USA. ${ }^{2}$ Amgen Inc., One Amgen Center Dr, Thousand Oaks, CA, USA. ${ }^{3}$ Simulstat, Inc., 4370 La Jolla Village Dr, San Diego, CA, USA. ${ }^{4}$ Amgen Inc., 1120 Veterans Blvd, South San Francisco, CA, USA. ${ }^{5}$ Onyx Pharmaceuticals, Inc., an Amgen subsidiary, 1641 Kansas St, Redwood City, CA, USA. ${ }^{6}$ NIHR Cardiovascular Biomedical Research Unit, Royal Brompton Hospital and Imperial College London, SW3 6NP, London, UK.

Received: 15 July 2016 Accepted: 10 November 2016

Published online: 22 November 2016

\section{References}

1. Anderson KC, Alsina M, Atanackovic D, Biermann JS, Chandler JC, Costello C, et al. NCCN Guidelines insights: Multiple myeloma, Version 3.2016. J Nat Compr Canc Netw. 2016;14(4):389-400.

2. SEER Cancer Statistics Factsheets. Myeloma. Bethesda: National Cancer Institute. http://seer.cancer.gov/statfacts/html/mulmy.html. Accessed 11 Feb 2016

3. Strait JD, Lakatta EG. Aging-associated cardiovascular changes and their relationship to heart failure. Heart Fail Clin. 2012;8(1):143-64

4. Quan H, Parsons GA, Ghali WA. Validity of information on comorbidity derived from ICD-9-CM administrative data. Med Care. 2002:40:675-85.

5. Dimopoulos MA, Terpos E, Niesvizky R, Palumbo A. Clinical characteristics of patients with relapsed multiple myeloma. Cancer Treat Rev. 2015;41(10): 827-35

6. Stewart AK, Rajkumar SV, Dimopoulos MA, Masszi T, Spicka I, Oriol A, et al. Carfilzomib, lenalidomide, and dexamethasone for relapsed multiple myeloma. N Engl J Med. 2015;372(2):142-52.

7. White D, Kassim A, Bhaskar B, Yi J, Wamstad K, Paton VE. Results from AMBER, a randomized phase 2 study of bevacizumab and bortezomib versus bortezomib in relapsed or refractory multiple myeloma. Cancer. 2013;119:339-47.

8. Kistler KD, Murphy B, Kalman J, Sahni G, Werther W, Rajangam K, et al. A comparison of cardiac event rates in patients with or without multiple myeloma in the United States. J Clin Oncol. 2014;32:e19563.

9. Nwankwo T, Yoon SS, Burt V, Gu Q. Hypertension among adults in the US: National Health and Nutrition Examination Survey, 2011-2012. NCHS Data Brief, No. 133. Hyattsville: National Center for Health Statistics, Centers for Disease Control and Prevention, US Dept of Health and Human Services; 2013.

10. Wong ND, Lopez VA, Italien GL, Chen R, Kline SEJ, Franklin SS. Inadequate control of hypertension in US adults with cardiovascular disease comorbidities in 2003-2004. Arch Intern Med. 2007;167(22):2431-36.

11. Chen YJ, De AP, Cong Z, Aggarwal SK, Wade RL. Demographic and comorbidity characteristics of newly diagnosed multiple myeloma patients in the United States: A real world data analysis. Blood. 2014;124(21):1301.

12. Sowers JR, Epstein M, Frohlich ED. Diabetes, hypertension, and cardiovascular disease. An update. Hypertension. 2001;37:1053-9.

13. James PA, Oparil S, Carter BL, Cushman WC, Dennison-Himmelfarb C, Handler J, et al. 2014 Evidence-based guidelines for the management of high blood pressure in adults. Report from the panel members appointed to the Eighth Joint National Committee (JNC8). JAMA. 2014;311(5):507-20.

14. Oshikawa G, Kojima A, Doki N, Kobayashi T, Kakihana K, Tsuda H, et al. Bortezomib-induced posterior reversible encephalopathy syndrome in a patients with newly diagnosed multiple myeloma. Intern Med. 2013:52:111-14.

15. Yang S, Jun M, Hong-Li Z, Jian-Min W, Chun W, Lu-Gui Q, et al. A multicenter open-labeled study of recombinant erythropoietin-beta in the treatment of anemic patients with multiple myeloma, low-grade nonHodgkin's lymphoma, or chronic lymphocytic leukemia in Chinese population. Int J Hematol. 2008:88(2):139-44.

16. Zhao $Y$, Jing $Y, B O J$, Li HH, Wang SH, Huang WR, et al. Adverse effects of PAD and VAD regimens in multiple myeloma patients. [Article in Chinese] Zhongguo Shi Yan Xue Ye Xue Za Zhi. 2010;18(4):1027-30.
17. Dimopoulos MA, Moreau P, Palumbo A, Joshua D, Pour L, Hájek R, et al. Carfilzomib and dexamethasone versus bortezomib and dexamethasone for patients with relapsed or refractory multiple myeloma (ENDEAVOR): a randomised, phase 3, open-label, multicentre study. Lancet Oncol. 2016; 17(1):27-38.

18. Papadopoulos KP, Siegel DS, Vesole DH, Lee P, Rosen ST, Zojwalla N, et al. Phase I study of 30-minute infusion of carfilzomib as single agent or in combination with low-dose dexamethasone in patients with relapsed and/ or refractory multiple myeloma. J Clin Oncol. 2015;33(7):732-9.

\section{Submit your next manuscript to BioMed Central and we will help you at every step:}

- We accept pre-submission inquiries

- Our selector tool helps you to find the most relevant journal

- We provide round the clock customer support

- Convenient online submission

- Thorough peer review

- Inclusion in PubMed and all major indexing services

- Maximum visibility for your research

Submit your manuscript at www.biomedcentral.com/submit
Biomed Central 